\title{
Exceptional family of elements for generalized variational inequalities
}

Xiao-lan Liu*

\section{"Correspondence:} stellalwp@163.com

School of Science, Sichuan University of Science and Engineering, Zigong, Sichuan 643000, China

\section{Springer}

\begin{abstract}
This paper introduces the notion of exceptional family of elements for generalized variational inequalities in Hilbert spaces. The set-valued mapping is assumed to be upper semi-continuous compact with nonempty closed convex values. Based on topological degree for set-valued mappings, instead of the technique of continuous selection, an alternative theorem is obtained which says that the generalized variational inequalities have either a solution or an exceptional family of elements. In addition, an existing result of a solution for generalized variational inequalities is obtained.

MSC: $90 C 25 ; 90 C 33$

Keywords: variational inequalities; exceptional family of elements; topological degree; upper semi-continuous; set-valued mapping
\end{abstract}

\section{Introduction}

Variational inequalities play an important role in nonlinear analysis. They have many important applications in different aspects such as mechanics, game theory, economics, optimization theory and nonlinear programming. How to solve the variational inequality problems has already become a basic problem, and then several classical methods have appeared. Several years ago, the introduction of an exceptional family of elements (exceptional family or $E F E$ for short) opened a new research direction in the solvability of variational inequality and complementarity problems. In the last four decades, many researchers have focused their efforts on finding the existence conditions for variational inequality and complementarity problems by virtue of the exceptional family of elements.

In 1984, Smith [1] introduced the exceptional sequence of elements to study the solution conditions for a single-valued complementarity problem in $\mathbb{R}^{n}$. The notion of exceptional family of elements was first used to study the solutions of variational inequality problems. From then on, the notion of exceptional family for complementarity problems has been generalized to set-valued mappings, or to more general spaces such as Hilbert spaces, Banach spaces, reflexive Banach spaces by several researchers. Some existence theorems for complementarity problems can be found in [2-5]. Zhao [6] proposed the notion of exceptional family of elements to solve a variational inequality problem. On the other hand, many researchers generalized this notion for variational inequality problems with a single-valued mapping defined in $\mathbb{R}^{n}$ to Hilbert spaces or normed spaces. For more details, see [7-10]. Applying their notions, they obtained some sufficient conditions for the solvability of variational inequality problems. Han et al. [7] established some sufficient

(c) 2013 Liu; licensee Springer. This is an Open Access article distributed under the terms of the Creative Commons Attribution License (http://creativecommons.org/licenses/by/2.0), which permits unrestricted use, distribution, and reproduction in any medium, provided the original work is properly cited. 
conditions for the solvability of variational inequality problems with single-valued continuous mappings in $\mathbb{R}^{n}$ and proved that those conditions were also necessary if the mapping was pseudomonotone. They also established some sufficient conditions for a quasimonotone variational inequality problem. Bianchi et al. [11] proved that the generalized complementarity problem either has a solution or an exceptional family of elements in Hilbert spaces. Under very weak assumptions on the mapping, Bianchi et al. [8] proposed a new exceptional family of elements for generalized variational inequality problems in normed spaces again. Their concept generalized those concepts proposed in [2] and [7]. They also obtained some existence theorems provided that the mapping was quasimonotone and upper sign-continuous. Very recently, Zhi et al. [12] introduced a new concept of exceptional family of elements for finite-dimensional generalized variational inequality problems and obtained an alternative theorem which says that the generalized variational inequality has either a solution or an exceptional family of elements. They also presented a sufficient condition to ensure the existence of a solution to the generalized variational inequality, and the set-valued mapping was assumed to be upper semi-continuous with nonempty compact convex values.

Inspired and motivated by the above research work, we apply the notion of exceptional family of elements for set-valued mappings in Isac sense [13] to a generalized variational inequality (in short, $\operatorname{GVI}(F, K)$ ) in Hilbert spaces. We prove that if the mapping is upper semi-continuous compact with nonempty closed convex values, then the generalized variational inequality has either a solution or an exceptional family of elements. And then, we obtain an existence result of a solution for $\operatorname{GVI}(F, K)$. Both above results are based on the topological degree theory for set-valued mappings, instead of the technique of continuous selection. For more details on topological degree, see [14]. Very recently, Jie et al. [15] studied the existence of solutions to a system of generalized order complementarity problems via an order exceptional family of elements. They proved that under certain conditions, the system of generalized order complementarity problems has either a solution or an order exceptional family of elements.

Our results extend the main results in $[4,12,16]$ and the references therein.

The organization of this paper is as follows. In the next section, we present some definitions and lemmas. We obtain an alternative theorem and an existence theorem of solution for $\operatorname{GVI}(F, K)$ in Section 3.

\section{Preliminaries}

For later discussion, some definitions and lemmas are introduced.

Let $H$ be an infinite dimensional Hilbert space whose inner product and norm are denoted by $\langle\cdot, \cdot\rangle$ and $\|\cdot\|$, respectively. Let $K$ be an unbounded closed convex subset of $H$ (without loss of generality, we assume that $x=0 \in K$ from now on), let $\Omega \subset H$ be an open bounded set such that $K \cap \Omega \neq \emptyset$. Let $F: K \rightarrow 2^{H}$ be a set-valued mapping. $F$ is said to be upper semi-continuous at $x \in K$ if for any open set $V \subset H$ such that $F(x) \subset V$, there exists an open neighborhood $U$ of $x$ such that $F(y) \subset V$ for all $y \in U \cap K$; if $F$ is upper semicontinuous at every $x \in K$, we say $F$ is upper semi-continuous on $K$. $F$ is said to be lower semi-continuous at $x \in K$ if for any open set $V \subset H$ such that $F(x) \cap V \neq \emptyset$, there exists an open neighborhood $U$ of $x$ such that $F(y) \cap V \neq \emptyset$ for all $y \in U \cap K$. F is lower semicontinuous at every $x \in K$, we say $F$ is lower semi-continuous on $K$. If $F$ is a single-valued mapping, both the definitions above provide the ordinary definition of continuity. 
Definition 2.1 A mapping $F: D(F) \rightarrow H$ is said to be compact if $F$ maps every bounded subset of $D(F)$ to a relatively compact subset in $H$. A set $M \subset H$ is said to be relatively compact if $\bar{M}$ is compact in $H$.

We recall that the generalized variational inequality problem $(G V I(F, K))$ is the following: find $x \in K$ and $x^{*} \in F(x)$ such that

$$
\left\langle x^{*}, y-x\right\rangle \geq 0, \quad \forall y \in K
$$

When $K$ is a cone, we get a particular case, which is the generalized complementarity problem $(G C P(F, K))$, as follows: find $x \in K$ and $x^{*} \in F(x)$ such that

$$
x^{*} \in K^{*} \quad \text { and } \quad\left\langle x, x^{*}\right\rangle=0 \text {, }
$$

where $K^{*}=\left\{x^{*} \in H:\left\langle x^{*}, x\right\rangle \geq 0, \forall x \in K\right\}$. If $F$ is single-valued mapping, then $G V I(F, K)$ reduces to $V I(F, K)$. In addition, if $K$ is a cone, then $V I(F, K)$ reduces to $C P(F, K)$.

Definition 2.2 Given a nonempty closed convex set $K$ in a Hilbert space $H$ and a setvalued mapping $F: K \rightarrow 2^{H}$, a family $\left\{x_{r}\right\}_{r>0} \subset K$ is an exceptional family of elements (in short, $E F E)$ for $F(x)=x-T(x)$ with respect to $K$ if

(i) $\left\|x_{r}\right\| \rightarrow \infty$ as $r \rightarrow \infty$;

(ii) for any $r>0$, there exist $\mu_{r}>1$ and $x_{r}^{*} \in T\left(x_{r}\right)$ such that $\mu_{r} x_{r} \in K$ and $x_{r}^{*}-\mu_{r} x_{r} \in N_{K}\left(\mu_{r} x_{r}\right)$.

Here, $N_{K}\left(x_{r}\right)$ is the normal cone to $K$ at the point $x_{r}$; i.e.,

$$
N_{K}\left(x_{r}\right)= \begin{cases}\left\{x^{*} \in K^{*}:\left\langle x^{*}, x-x_{r}\right\rangle \leq 0, \forall x \in K\right\} & \text { if } x_{r} \in K, \\ \varnothing & \text { if } x_{r} \in H \backslash K .\end{cases}
$$

It is easy to see that $\lambda N_{K}\left(x_{r}\right)=N_{K}\left(x_{r}\right)$ for any $\lambda \geq 0$, it means that $N_{K}\left(x_{r}\right)$ is a cone.

Now we propose another definition of an exceptional family of elements. Throughout this paper, we use the following definition of an exceptional family of elements.

Definition 2.3 $\left\{x_{r}\right\}_{r>0} \subset K$ is an exceptional family of elements of $G V I(F, K)$ if

(i) $\left\|x_{r}\right\| \rightarrow \infty$ as $r \rightarrow \infty$;

(ii) for any $r>0$, there exist $t_{r} \in(0,1)$ and $x_{r}^{*} \in F\left(x_{r}\right)$ such that

$$
-t_{r} x_{r}-\left(1-t_{r}\right) x_{r}^{*} \in N_{K}\left(x_{r}\right) .
$$

The following lemma formulates an important property of the exceptional family of elements.

Lemma 2.1 Assume that $\left\{x_{r}\right\}_{r>0} \subset K$ is an exceptional family of elements of $G V I(F, K)$ for any $x_{r} \neq 0$, then $\left\langle x_{r}^{*}, x_{r}\right\rangle<0, x_{r}^{*} \in F\left(x_{r}\right)$.

Proof By the definition of $N_{K}(x)$ and (3), we have

$$
\left\langle-t_{r} x_{r}-\left(1-t_{r}\right) x_{r}^{*}, y-x_{r}\right\rangle \leq 0, \quad \forall y \in K, r>0, x_{r}^{*} \in F\left(x_{r}\right)
$$


i.e.,

$$
\left\langle\left(1-t_{r}\right) x_{r}^{*}, y-x_{r}\right\rangle \geq-t_{r}\left\langle x_{r}, y-x_{r}\right\rangle, \quad \forall y \in K, r>0, x_{r}^{*} \in F\left(x_{r}\right),
$$

in which we take $y=0$ and it follows that $\left\langle x_{r}^{*}, x_{r}\right\rangle \leq-\frac{t_{r}}{1-t_{r}}\left\|x_{r}\right\|^{2}<0, \forall x_{r} \neq 0, x_{r}^{*} \in F\left(x_{r}\right)$. The proof is complete.

Remark 2.1 From the proof of Lemma 2.1, we see that (4) means that $x_{r}$ is a solution of the generalized variational inequality. Hence, the existence of exceptional family is a problem as difficult as the existence of $G V I(F, K)$. However, we discuss not the existence of an exceptional family, but the nonexistence of the exceptional family which implies the existence of the solution of $G V I(F, K)$.

Remark 2.2 Assume that $\left\{x_{r}\right\}_{r>0} \subset K$ is an exceptional family defined by Definition 2.2, then there exist $\mu_{r}>1$ and $x_{r}^{*} \in F\left(x_{r}\right)$ such that $\mu_{r} x_{r} \in K$ and

$$
x_{r}-x_{r}^{*}-\mu_{r} x_{r} \in N_{K}\left(\mu_{r} x_{r}\right),
$$

i.e.,

$$
-\left(1-\mu_{r}^{-1}\right) x_{r}-\mu_{r}^{-1} x_{r}^{*} \in \mu_{r}^{-1} N_{K}\left(\mu_{r} x_{r}\right)
$$

If $N_{K}\left(\mu_{r} x_{r}\right)=\mu_{r} N_{K}\left(x_{r}\right)$, then (5) can be rewritten as

$$
-t_{r} x_{r}-\left(1-t_{r}\right) x_{r}^{*} \in N_{K}\left(x_{r}\right)
$$

where $t_{r}=1-\mu_{r}^{-1}$. Obviously, (6) means $\left\{x_{r}\right\}_{r>0} \subset K$ is an exceptional family defined by Definition 2.3.

From the discussion above, we have the following lemma.

Lemma 2.2 Assume that $\mu K \subset K$ and $N_{K}(\mu x)=\mu N_{K}(x)$ for any $\mu>1, x \in K$. Then Definition 2.2 is equivalent to Definition 2.3.

Remark 2.3 Obviously, if $H=\mathbb{R}^{n}$ and $K=\mathbb{R}_{+}^{n}$, then $K$ satisfies the condition of Lemma 2.2.

Now we consider a particular case: $H=\mathbb{R}^{n}$ and $K$ is defined as follows:

$$
K=\left\{x \in \mathbb{R}^{n}: g_{i}(x) \leq 0, i=1, \ldots, I ; h_{i}(x)=0, j=1, \ldots, J\right\},
$$

where $g_{i}: \mathbb{R}^{n} \rightarrow R$ is a continuously differentiable convex function and $h_{j}$ is an affine function. Furthermore, we assume that $K$ satisfies the Slater condition, i.e., there is a point $x_{0} \in K$ such that $g_{i}\left(x_{0}\right)<0, i=1, \ldots, I$. Zhao et al. [17] defined an exceptional family of elements as follows.

Definition 2.4 $\left\{x_{r}\right\}_{r>0} \subset K$ which is defined by (7) is an exceptional family of elements for $G V I(F, K)$ if 
(i) $\left\|x_{r}\right\| \rightarrow \infty$ as $r \rightarrow \infty$;

(ii) for any $r>0$, there exist $\alpha_{r}>0, \lambda_{r} \in \mathbb{R}_{+}^{I}$ and $\mu_{r} \in \mathbb{R}^{J}, x_{r}^{*} \in F\left(x_{r}\right)$ such that

$$
x_{r}^{*} \in-\alpha_{r} x_{r}-\frac{1}{2}\left[\sum_{i=1}^{I} \lambda_{r_{i}} \nabla g_{i}\left(x_{r}\right)+\sum_{j=1}^{J} \mu_{r_{j}} \nabla h_{j}(x)\right], \quad \sum_{i=1}^{I} \lambda_{r_{i}} g_{i}\left(x_{r}\right)=0
$$

It is well known that for $K$ defined by (7), we have that

$$
N_{K}(x)=\left\{z=\sum_{1=1}^{I} \lambda_{i} \nabla g_{i}(x)+\sum_{j=1}^{J} \mu_{r} \nabla h_{j}\left(x_{r}\right): \lambda \in \mathbb{R}_{+}^{I} \text { and } \mu \in \mathbb{R}^{J}, \sum_{i=1}^{I} \lambda_{i} g_{i}(x)=0\right\} \text {. }
$$

It follows from (8) and (9),

$$
-2 \alpha_{r} x_{r}-2 x_{r}^{*} \in N_{K}\left(x_{r}\right), \quad x_{r}^{*} \in F\left(x_{r}\right) .
$$

With $\alpha=2\left(\alpha_{r}+1\right)$ and $t_{r}=2 \alpha^{-1} \alpha_{r}$, we have that $t_{r} \in(0,1)$ and $1-t_{r}=2 \alpha^{-1}$. Hence, (10) can be written as

$$
-t_{r} x_{r}-\left(1-t_{r}\right) x_{r}^{*} \in \alpha^{-1} N_{K}\left(x_{r}\right)=N_{K}\left(x_{r}\right)
$$

Therefore, we have the following result.

Lemma 2.3 If $K$ is defined by (7), Definition 2.3 is equivalent to Definition 2.4.

Lemma 2.4 The projection operator $P_{K}$ is characterized by the following result:

$$
\left\langle P_{K}(x)-x, y-P_{K}(x)\right\rangle \geq 0, \quad \forall y \in K
$$

Lemma $2.5 x$ is a solution of $\operatorname{GVI}(F, K)$ if and only if $x$ is the solution of the nonlinear equation

$$
0 \in x-P_{K}(x-F(x))
$$

Next, we recall the definition of topological degree for set-valued mappings (denoted by $\operatorname{deg}(\cdot))$ in $[14]$.

Definition 2.5 Let $E$ be a real Banach space, $\Omega \subset E$ be an open bounded set and $T: \bar{\Omega} \rightarrow$ $2^{E}$ be an upper semi-continuous mapping with closed convex values. Suppose that $T \bar{\Omega}$ is relatively compact and $x \notin T x$ for all $x \in \partial \Omega$. Then we define

$$
\operatorname{deg}(I-T, \Omega, 0)=\lim _{\epsilon \rightarrow 0} \operatorname{deg}\left(I-f_{\epsilon}, \Omega, 0\right),
$$

where $f_{\epsilon}$ is defined as in Lemma 2.3.7 in [14].

The following property follows from Definition 2.5 which is Theorem 2.3.13 in [14].

Lemma 2.6 The degree defined by Definition 2.5 has the following properties:

(1) (Normality) $\operatorname{deg}(I, \Omega, 0)=1$ if and only if $0 \in \Omega$; 
(2) (Solvability) If $\operatorname{deg}(I-T, \Omega, 0) \neq 0$, then $x \in T x$ has a solution in $\Omega$;

(3) (Homotopy) Let $T_{t}:[0,1] \times \bar{\Omega} \rightarrow$ E be an upper semi-continuous compact mapping with closed convex values and $x \notin T_{t} x$ for all $(t, x) \in[0,1] \times \partial \Omega$. Then $\operatorname{deg}\left(I-T_{t}, \Omega, 0\right)$ does not depend on $t \in[0,1]$;

(4) (Additivity) If $\Omega_{1}, \Omega_{2}$ are two disjoint open subsets of $\Omega$ and $0 \notin(I-T)\left(\bar{\Omega}-\Omega_{1} \cup \Omega_{2}\right)$, then

$$
\operatorname{deg}(I-T, \Omega, 0)=\operatorname{deg}\left(I-T, \Omega_{1}, 0\right)+\operatorname{deg}\left(I-T, \Omega_{2}, 0\right) .
$$

\section{Exceptional family of elements}

The following conclusions are useful in the proof of the main result of our work, which generalizes the corresponding result of [12] from a finite dimensional space $\mathbb{R}^{n}$ to an infinite dimensional Hilbert space $H$.

Throughout this section, let $H$ be a Hilbert space and let $K$ be a closed convex subset of $H$.

Theorem 3.1 For any set-valued mapping $F: K \rightarrow 2^{H}$ such that $F(x)=x-G(x)$, where $G: H \rightarrow 2^{H}$ is an upper semi-continuous compact mapping with nonempty closed convex values, then there exists a solution of $G V I(F, K)$ or an exceptional family of elements for $\operatorname{GVI}(F, K)$.

Proof For any $r>0$, we denote

$$
B_{r}=\{x \in H \mid\|x\|<r\}, \quad S_{r}=\partial B_{r}=\{x \in H \mid\|x\|=r\},
$$

which is such that $B_{r} \cap K \neq \emptyset$ and $S_{r} \cap K \neq \emptyset$. By Lemma 2.5 , we know that the solvability of $G V I(F, K)$ is equivalent to the equation $0 \in x-P_{K}(x-F(x))$ is solvable in $K$. Let $T(x)=$ $x-P_{K}(x-F(x))$ and

$$
h(x, t)=t x+(1-t) T(x) .
$$

From the definition of $T$, we have

$$
\begin{aligned}
h(x, t) & =t x+(1-t)\left[x-P_{K}(x-F(x))\right] \\
& =x-(1-t) P_{K}(x-F(x)) \\
& =x-H(x, t),
\end{aligned}
$$

where $H(x, t)=(1-t) P_{K}(x-F(x))=(1-t) P_{K}(G(x)), h(x, 0)=T(x)=x-P_{K}(x-F(x))$, $h(x, 1)=x$. Since $G$ is upper semi-continuous compact with nonempty closed convex values, $P_{K}$ is continuous, thus $H$ is upper semi-continuous compact with nonempty closed convex values. It also shows that $h: \overline{B_{r}} \times[0,1] \rightarrow 2^{H}$ is upper semi-continuous compact with nonempty closed convex values. Since $0 \in B_{r}$, by (1) of Lemma 2.6, thus $\operatorname{deg}\left(I, B_{r}, 0\right)=1$.

By using the topological degree for set-valued mappings and applying Theorem 2.3.13 in [14], we have the following two cases: 
(1) there exists an $r>0$ such that

$0 \notin h(x, t), \quad$ i.e. $, x \notin H(x, t), \forall x \in S_{r}, \forall t \in[0,1]$.

By (3) of Lemma 2.6, $\operatorname{deg}\left(h(x, 0), B_{r}, 0\right)=\operatorname{deg}\left(h(x, 1), B_{r}, 0\right)$, i.e., $\operatorname{deg}\left(T, B_{r}, 0\right)=\operatorname{deg}\left(I, B_{r}, 0\right)$. It follows that $\operatorname{deg}\left(T, B_{r}, 0\right)=\operatorname{deg}\left(I, B_{r}, 0\right)=1$. Thus, $0 \in x-P_{K}(x-F(x))$. From Lemma 2.5, then $\operatorname{GVI}(F, K)$ has a solution in $B_{r}$.

(2) For each $r>0$, there exist a point $x_{r} \in S_{r}$ and a scalar $t_{r} \in[0,1]$ such that

$$
0 \in h\left(x_{r}, t_{r}\right) \text {, i.e., } x_{r} \in H\left(x_{r}, t_{r}\right) \text {. }
$$

We now claim that $t_{r} \neq 0$ and $t_{r} \neq 1$. In fact, if $t_{r}=0$, then from (13) we have that $0 \in h\left(x_{r}, 0\right)$, that is, $x_{r} \in H\left(x_{r}, 0\right)$, i.e., $0 \in T\left(x_{r}\right)$, then $0 \in x_{r}-P_{K}\left(x_{r}-F\left(x_{r}\right)\right)$. From Lemma 2.5, then $G V I(F, K)$ has a solution. If $t_{r}=1$, then from (13) and the definition of $T(x)$ and $h(x, t)$, it follows that $0 \in h\left(x_{r}, 1\right)$, i.e., $x_{r}=0$, which is impossible since $x_{r} \in S_{r}$. So, we can say that $G V I(F, K)$ has a solution, or for any $r>0$, there exist a point $x_{r} \in S_{r}$ and $t_{r} \in(0,1)$ such that $0 \in h\left(x_{r}, x_{r}\right)$. From (11), we deduce that

$$
0 \in x_{r}-\left(1-t_{r}\right) P_{K}\left(x_{r}-F\left(x_{r}\right)\right)
$$

or

$$
\frac{1}{1-t_{r}} x_{r} \in P_{K}\left(x_{r}-F\left(x_{r}\right)\right)
$$

i.e.,

$$
x_{r} \in P_{K}\left(\left(1-t_{r}\right)\left(x_{r}-F\left(x_{r}\right)\right)\right) .
$$

By Lemma 2.4, we deduce that there exists $x_{r}^{*} \in F\left(x_{r}\right)$ such that

$$
\left\langle x_{r}-\left(1-t_{r}\right)\left(x_{r}-x_{r}^{*}\right), y-x_{r}\right\rangle \geq 0, \quad \forall y \in K .
$$

It follows that

$$
x_{r}-\left(1-t_{r}\right)\left(x_{r}-x_{r}^{*}\right) \in-N_{K}\left(x_{r}\right) \text {, }
$$

i.e.,

$$
t_{r} x_{r}+\left(1-t_{r}\right) x_{r}^{*} \in-N_{K}\left(x_{r}\right) \text {. }
$$

It follows that

$$
-t_{r} x_{r}-\left(1-t_{r}\right) x_{r}^{*} \in N_{K}\left(x_{r}\right) \text {. }
$$

Since for each $r>0$ there exists a point $x_{r} \in S_{r}$, from (14), $x_{r} \in P_{K}\left(\left(1-t_{r}\right)\left(x_{r}-F\left(x_{r}\right)\right)\right)$, we obtain that $x_{r} \in K$. On the other hand, $x_{r} \in S_{r}$, it follows that $\left\|x_{r}\right\|=r$, and then $\left\|x_{r}\right\| \rightarrow \infty$ as $r \rightarrow \infty$. Therefore, we know that $\left\{x_{r}\right\}_{r>0}$ is an exceptional family of $G V I(F, K)$. This completes the proof. 
Remark 3.1 As an application of exceptional family of elements for set-valued mappings in Isac sense, in Theorem 3.1, we establish the alternative theorem of the existence of a solution for $\operatorname{GVI}(F, K)$ based on the topological degree for set-valued mappings, instead of the Leray Schauder type alternative and the technique of continuous selection. As a consequence, the method used in Theorem 3.1 is quite different from that in [13] and [18]. When $F$ is quasimonotone and upper sign-continuous with nonempty $w^{*}$-compact and convex values, and $K$ is a closed convex cone in a Hilbert space, the result is presented in [11]. When $H=\mathbb{R}^{n}, F$ is upper semi-continuous set-valued with nonempty compact convex values, the result is presented in [12]. When $K$ is a closed convex cone, Theorem 3.1 reduces to Theorem 3.1 in [4].

From Theorem 3.1, we have the following result.

Theorem 3.2 For any set-valued mapping $F: K \rightarrow 2^{H}$ such that $F(x)=x-G(x)$, where $G: H \rightarrow 2^{H}$ is an upper semi-continuous compact mapping with nonempty closed convex values, if there does not exist an exceptional family of elements for $G V I(F, K)$, then there exists a solution of $G V I(F, K)$.

Corollary 3.1 Let $K$ be a nonempty closed convex set of $H$. If a mapping $F: K \rightarrow H$ is such that $F(x)=x-G(x)$, where $G: H \rightarrow H$ is a continuous compact mapping with nonempty closed convex values, then there exists a solution of $V I(F, K)$ or an exceptional family of elements for $(\operatorname{VI}(F, K))$.

Corollary 3.2 Let $H$ be a Hilbert space, let $K$ be a closed convex cone of $H$. For any setvalued mapping $F: K \rightarrow 2^{H}$ such that $F(x)=x-G(x)$, where $G: H \rightarrow 2^{H}$ is an upper semicontinuous compact mapping with nonempty closed convex values, there exists a solution of $\operatorname{GCP}(F, K)$ or an exceptional family of elements for $G C P(F, K)$.

Corollary 3.3 Let $H$ be a Hilbert space, let $K$ be a closed convex cone of $H$. If a mapping $F: K \rightarrow H$ is such that $F(x)=x-G(x)$, where $G: H \rightarrow H$ is a continuous compact mapping with nonempty closed convex values, then there exists a solution of $C P(F, K)$ or an exceptional family of elements for $C P(F, K)$.

\section{Competing interests}

The author declares that they have no competing interests.

\section{Acknowledgements}

This work is partially supported by the National Natural Science Foundation of China (Grant No. 11126336), the Scientific Research Fund of Sichuan Provincial Education Department (12ZA098), the Scientific Research Fund of Sichuan University of Science and Engineering (2012KY08).

Received: 14 September 2012 Accepted: 17 June 2013 Published: 12 July 2013

References

1. Smith, TE: A solution condition for complementarity problems, with an application to spatial price equilibrium. Appl. Math. Comput. 15, 61-69 (1984)

2. Isac, G, Kalashnikov, V: Exceptional family of elements, Leray-Schauder alternative, pseudomonotone operators, and complementarity. J. Optim. Theory Appl. 109, 69-83 (2001)

3. Isac, G, Li, J: Exceptional family of elements and the solvability of complementarity problems in uniformly smooth and uniformly convex Banach spaces. J. Zhejiang Univ. Sci. 6A, 289-295 (2005)

4. Li, J, Huang, NJ: Exceptional family of elements for set-valued mappings: an application to nonlinear complementarity problems. Appl. Math. Lett. 21, $42-46$ (2008) 
5. Zhao, YB, Yuan, JY: An alternative theorem for generalized variational inequalities and solvability of nonlinear quasi- $P_{*}^{M}$-complementarity problems. Appl. Math. Comput. 109, 167-182 (2000)

6. Zhao, YB: Exceptional family and finite-dimensional variational inequality over polyhedral convex set. Appl. Math Comput. 87, 111-126 (1997)

7. Han, J, Huang, ZH, Fang, SC: Solvability of variational inequality problems. J. Optim. Theory Appl. 122, 501-520 (2004)

8. Bianchi, M, Hadjisavvas, N, Schaible, S: Exceptional family of elements for variational inequalities in Banach spaces. J. Optim. Theory Appl. 129, 23-31 (2006)

9. Isac, G, Zhao, YB: Exceptional family of elements and solvability of variational inequalities for unbounded sets in infinite-dimensional Hilbert spaces. J. Math. Anal. Appl. 246, 544-556 (2000)

10. $L i, J$, Whitaker, J: Exceptional family of elements and solvability of variational inequalities for mapping defined only on closed convex cones in Banach spaces. J. Math. Anal. Appl. 310, 254-261 (2005)

11. Bianchi, M, Hadjisavvas, N, Schaible, S: Minimal coercivity conditions and exceptional family of elements in quasimonotone variational inequalities. J. Optim. Theory Appl. 122, 1-17 (2004)

12. Zhi, L, Yiran, H: Exceptional family of elements for generalized variational inequalities. J. Glob. Optim. 48, 465-471 (2010)

13. Isac, G: Topological Methods in Complementarity Theory. Kluwer Academic, Dordrecht (2000)

14. Donal, O, Yeol, JC, Yu, QC: Topological Degree Theory and Application. Taylor \& Francis, Boca Raton (2006)

15. Zhao, J, Hu, R, Fang, YP: An existence result for a system of generalized order complementarity problems via order exceptional families of elements. Positivity (2013). doi:10.1007/s11117-013-0230-z

16. Isac, G: Exceptional family of elements, feasibility and complementarity. J. Optim. Theory Appl. 3, 577-588 (2000)

17. Zhao, YB, Han, JY, Qi, HD: Exceptional families and existence theorems for variational inequality problems. J. Optim. Theory Appl. 101, 475-495 (1999)

18. Kalashnikov, W, Isac, G: Solvability of implicit complementarity problems. Ann. Oper. Res. 116, 199-221 (2002)

doi:10.1186/1029-242X-2013-321

Cite this article as: Liu: Exceptional family of elements for generalized variational inequalities. Journal of Inequalities and Applications 2013 2013:321.

\section{Submit your manuscript to a SpringerOpen ${ }^{\circ}$ journal and benefit from:}

$\rightarrow$ Convenient online submission

- Rigorous peer review

- Immediate publication on acceptance

- Open access: articles freely available online

- High visibility within the field

- Retaining the copyright to your article 